\title{
Successful management of absent sternum in an infant using porcine acellular dermal matrix
}

\author{
Roy Alfred Semlacher ${ }^{1}$, Muhammand A.K. Nuri ${ }^{2}$ \\ ${ }^{1}$ Department of Plastic and Reconstructive Surgery, Mary Bridge Children's Hospital \& Health Network, MultiCare Health System, Tacoma, \\ WA; ${ }^{2}$ Seattle Children's and Mary Bridge Children's Regional Cardiac Surgery Program, Tacoma, WA, USA
}

Congenital absent sternum is a rare birth defect that requires early intervention for optimal long-term outcomes. Descriptions of the repair of absent sternum are limited to case reports, and no preferred method for management has been described. Herein, we describe the use of porcine acellular dermal matrix to reconstruct the sternum of an infant with sternal infection following attempted repair using synthetic mesh. The patient was a full-term male with trisomy 21, agenesis of corpus callosum, ventricular septal defect, patent ductus arteriosus, right-sided aortic arch, and congenital absence of sternum with no sternal bars. Following removal of the infected synthetic mesh, negative pressure wound therapy with instillation was used to manage the open wound and provide direct antibiotic therapy. When blood Creactive protein levels declined to $\leq 2 \mathrm{mg} / \mathrm{L}$, the sternum was reconstructed using porcine acellular dermal matrix. At 21 months postoperative, the patient demonstrated no respiratory issues. Physical examination and computed tomography imaging identified good approximation of the clavicular heads and sternal cleft and forward curvature of the ribs. This case illustrates the benefits of negative pressure wound therapy and acellular dermal matrix for the reconstruction of absent sternum in the context of infected sternal surgical site previously repaired with synthetic mesh.

Keywords Sternum / Acellular dermis / Surgical mesh / Thoracic surgery / Negative pressure wound therapy

\author{
Correspondence: \\ Roy Alfred Semlacher \\ Department of Plastic and \\ Reconstructive Surgery, Mary Bridge \\ Children's Hospital, 316 Martin Luther \\ King Jr. Way, Suite 314, Tacoma, WA \\ 98405, USA \\ Tel: +1-253-403-2120 \\ Fax: +1-253-403-2654 \\ E-mail: rsemlacher@multicare.org
}

The authors thank Tyler Seick (Mary Bridge Children's Hospital, Tacoma, WA, USA) for assist of surgery.

Received: July 10, 2018 • Revised: October 26, 2018 - Accepted: December 15, 2018

pISSN: 2234-6163 • elSSN: 2234-6171 • https://doi.org/10.5999/aps.2018.00829 • Arch Plast Surg 2019;46:470-474

\section{INTRODUCTION}

Complete congenital sternal cleft, also called absent sternum, is a very rare and potentially life-threatening birth defect. The condition involves the lack of bone between hyoid and pubis and often co-occurs with a variety of other abnormalities of midline fusion $[1,2]$. The absence of a sternum introduces multiple risks to the patient, including mediastinal injury, hypothermia, insensible fluid losses, and respiratory and hemodynamic sequelae [1]. The condition can be diagnosed prenatally or at birth. Early intervention is preferred, while the thoracic cage remains compliant, to protect the heart and mediastinal structures and support thoracic function. Long-term management may require further reconstruction with autologous tissues to support normal growth.

Published experience with absent sternum is limited to sparse case reports, and no preferred method for management has been described $[1,2]$. In this report, we describe the use of acellular dermal matrix $(\mathrm{ADM})$ to manage absent sternum in a fullterm infant, despite infection of previously placed synthetic 
mesh. Sternal reconstruction, in the presence of infection, was required because the patient could not be weaned from highflow oxygen due to unstable pulmonary mechanics.

\section{CASE}

Consent to publish the case report was obtained from the patient's family. This report does not contain any personal information that could lead to the identification of the patient.

The patient was a full-term male with a diagnosis of trisomy 21 , agenesis of corpus callosum, small restrictive ventricular septal defect, large patent ductus arteriosus (PDA), right-sided aortic arch, and congenital absence of sternum with no sternal bars (Fig. 1). Follow-up echocardiogram demonstrated spontaneous closure of the PDA.

On day 10 post-birth, the patient underwent sternal stabilization with a synthetic repair material (Physiomesh; Ethicon, Bridgewater, NJ, USA), which was sutured to ribs 2 to 5 bilaterally and the superior aspect of the rectus abdominus. Bilateral pectoralis major flaps were elevated. Because the left pectoralis major was insufficient to cross the midline, a bovine pericardial patch was used to bridge the medial borders of the pectoralis muscles.

Initially, the patient responded well. However, on postoperative day (POD) 13, the patient developed a clinical infection with fluctuation over the left anterior shoulder. Cultures identified methicillinsensitive Staphylococcus aureus (MSSA). Infectious disease consult initiated vancomycin and piperacillin/ tazobactam and recommended removal of the synthetic mesh used for sternal reconstruction.

On POD 21, the synthetic mesh and bovine pericardium were removed and mediastinal exploration was performed. Negativepressure wound therapy using the V.A.C. (KCI; Acelity Co., San Antonio, TX, USA) was used to manage and protect the open

\section{Fig. 1. Thoracic CT showing absent sternum before reconstruction}

(A) Computed tomography (CT) and (B) 3-dimensional reconstruction of patient's thoracic cage at age 3 days, before sternal reconstruction. Note absence of sternum and open shape of thoracic cage, with left and right ribs in parallel, rather than curved.
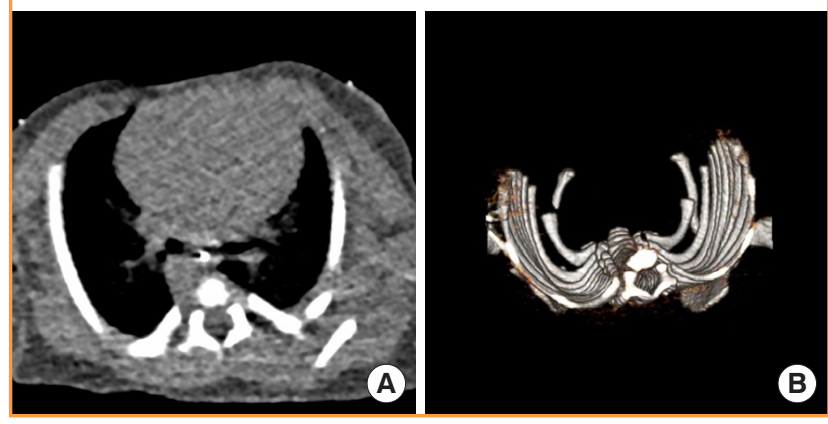

wound. Intraoperative cultures were positive for MSSA.

On POD 24, the patient was taken back to operating room and negative-pressure wound therapy was applied using the V.A.C. VERAFLO (KCI; Acelity Co.), which has both negative-pressure and instillation capabilities. The device was programmed to instill $6 \mathrm{~mL}$ of a rifampin-gentamycin solution, which was left in place for 5 minutes and then evacuated, followed by re-initiation of negative pressure therapy. This cycle was repeated every 4 hours. At this time, serum C-reactive protein (CRP) was $6.0 \mathrm{mg} / \mathrm{L}$.

On POD 30, the patient underwent sternal reconstruction with rifampin-impregnated porcine ADM (Strattice; Allergan plc, Madison, NJ, USA). A surgical plane was developed between the rib heads and the pericardium on both sides. Bilateral pectoralis major myocutaneous were raised laterally to the midclavicular level. Sutures (4.0 PDS-plus) were then placed in the ribs and through the ADM in a horizontal mattress fashion, placing the $\mathrm{ADM}$ in an underlay position between the pericardium and the ribs. The bilateral pectoralis major myocutaneous flaps were readvanced and approximated in the midline with 4.0 PDS-plus sutures placed at the muscle fascia-subcutaneous junction. By POD 45, all antibiotics were discontinued; CRP level was $0.3 \mathrm{mg} / \mathrm{L}$. The patient was discharged on POD 51 .

At 21 months POD, the patient demonstrated no clinical concerns, including no respiratory issues and was requiring no supplemental oxygen. The infant was sitting but had not started crawling. Examination identified a $1.5-\mathrm{cm}$ gap between the clavicular heads at the superior aspect of the sternum, with good approximation of the sternal cleft in the inferior aspect. Computed tomography imaging, 9 months post-sternal reconstruction, demonstrated forward curvature of ribs as would occur normally in the presence of a sternum (Fig. 2). Plain photography at 3 years after the ADM procedure showed full healing and normal chest development (Fig. 3).

\section{Fig. 2. Thoracic CT 9 months after sternal reconstruction}

(A) Computed tomography (CT) image and (B) 3-dimensional reconstruction of patient's thoracic cage 9 months after sternal reconstruction with acellular dermal matrix. Note normal anterior curvature of ribs, as would occur in the presence of a sternum.
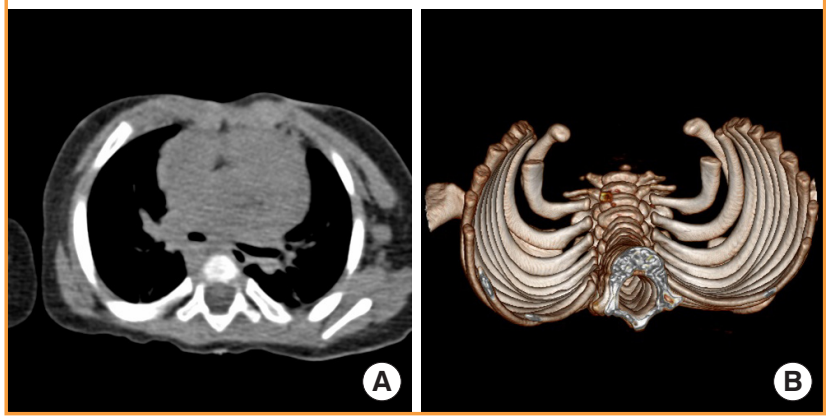


\section{Fig. 3. Patient at 3 years after sternal reconstruction}

Photograph of patient 3 years after acellular dermal matrix-based sternal reconstruction. The wound has fully healed and the thoracic cage shows normal structure and development.

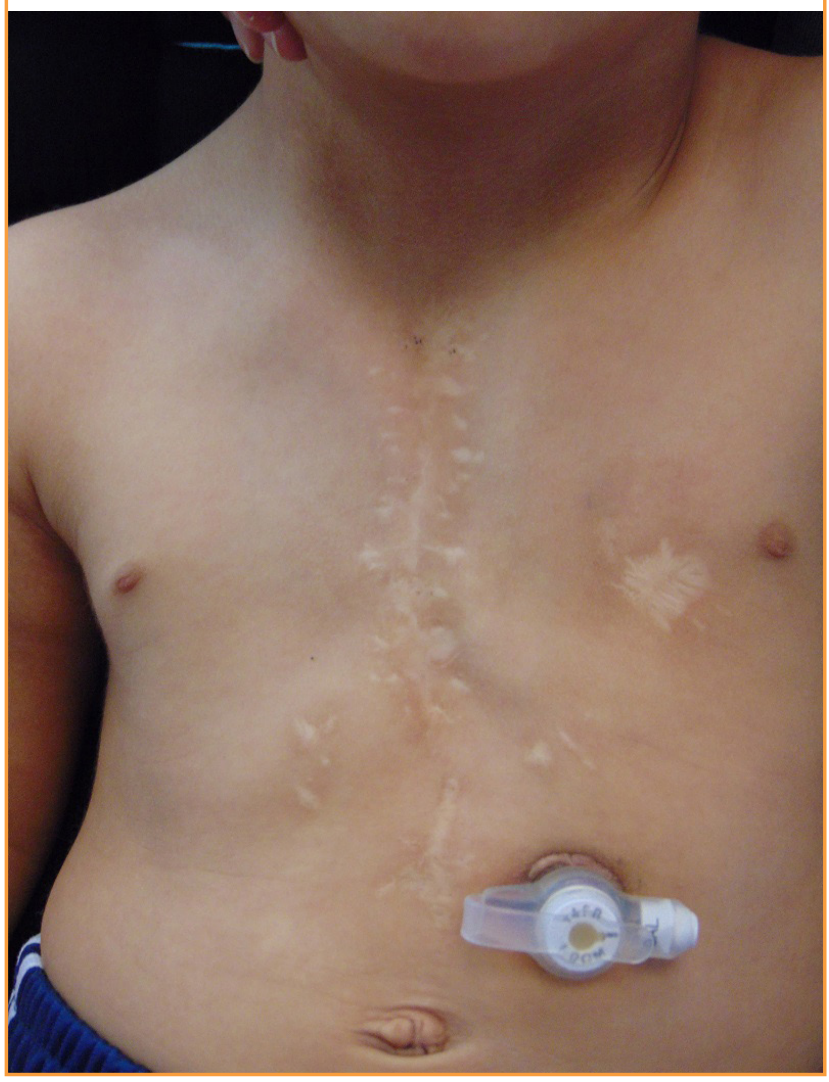

\section{DISCUSSION}

Complete sternal cleft, also called absent sternum, is an uncommon form of the rare congenital sternal cleft abnormalities. Although absent sternum can occur in isolation, it is often associated with a variety of other midline abnormalities, such as Cantrell's pentalogy. In the current case, the patient had multiple congenital abnormalities, including trisomy 21 , agenesis of corpus callosum, a small restrictive ventricular septal defect, rightsided aortic arch, and a large PDA, which resolved spontaneously. Repair of the sternal deficit in patients with absent sternum is required to protect mediastinal structures and support thoracic function.

The description and management of absent sternum is limited to case reports in the literature. Reported methods of repair include primary approximation (i.e., suturing the proximal ends of the ribs across the midline), sliding or rotating chondrotomies, and reconstructions using autogenous tissues or prosthetic repair materials [2-4]. Because of age-related changes in thoracic compliance and advances in neonatal surgery, correction of the sternal deficit should be attempted as early as possible. In neonates, for example, the sternal bars (if present) can easily be approximated with simple sutures, as the thoracic cage remains highly compliant at this age. However, the complete absence of sternal bars, as in the current case, may dictate the need for more advanced techniques to achieve closure of the thorax.

Previous case reports have described the use of synthetic repair materials to repair partial or complete sternal clefts [4-6]. In the current case, initial sternal reconstruction was attempted using a composite synthetic mesh (Physiomesh) composed of a nonabsorbable, macroporous polypropylene mesh laminated between two layers of absorbable mesh. The advantages of synthetic repair materials have been described in other fields of surgery, most notably hernia repair. These materials are relatively inexpensive, demonstrate good tensile strength, and provide permanent (nonabsorbable mesh) or temporary (absorbable mesh) support for repair of tissue deficits. However, synthetic repair materials typically require removal in the setting of infection and are often avoided in patients at high risk for infection [7-9]. The synthetic mesh used in this case became infected with MSSA and required removal. Previous case studies have also reported the removal of infected synthetic mesh following repair of cleft sternum [6].

Unlike synthetic mesh, biologic repair materials, including the porcine $\mathrm{ADM}$ used in this case, consist of extracellular matrix (ECM) derived from biologic tissues, which fosters remodeling of the prosthesis. The ECM in these materials supports the migration of native cells into the prosthesis, the ingrowth of blood vessels, and incorporation of the prosthesis into the surgical site. Of relevance to the current case, the high level of infiltration of biologic repair materials by blood vessels and immune cells means that these prostheses often can be managed without removal in the setting of infection and are generally preferred for cases with high infection risk [7-11].

The current case illustrates these advantages of biologic repair materials. When the synthetic repair material became infected, the mesh was fully removed and the wound was managed with negative pressure wound therapy (V.A.C.), followed by a device that provides both negative-pressure wound therapy and antibiotic instillation into the wound (V.A.C. VERAFLO). These devices create a negative-pressure environment at the wound site, which holds the incision edges together, reduces lateral tension and edema, stimulates perfusion, enhances the development of granulation tissue, reduces bacterial colonization of wound tissues, protects the surgical site from external infectious sources, and prepares the wound surface for the acceptance of skin grafts when needed [12-14]. The use of negative-pressure wound therapy for the management of deep sternal wounds is a relatively new technique that has been associated with good out- 
comes in adults following sternotomy [15]. The benefit of the V.A.C. VERAFLO in the current case were 2-fold. First, it stabilized the chest wall anteriorly, mimicking manubrial-clavicular stability and improving the patient's pulmonary function as a temporizing measure while preparing for reconstruction with $\mathrm{ADM}$. Second, it allowed direct delivery of antibiotics to the infected wound, analogous to the antibiotics beads often used in orthopedics. There were no concerns of having the V.A.C. in place over the heart because the pericardium was intact.

The reason that $\mathrm{ADM}$ reconstruction was attempted despite deep sternal infection was that the patient required high-flow oxygen due to unstable pulmonary mechanics. Reconstruction was urgently needed to stabilize the patient's pulmonary function. The long-term benefits of ADM reconstruction in this case include the reapproximation of the rib heads and support for the development of natural rib curvature. Because of this reapproximation of normal anatomy, autologous tissues may be used if additional sternal reconstruction is required to support further growth and development, potentially simplifying future procedures.

Following the application of negative pressure wound therapy and direct wound irrigation with antibiotics (along with systemic antibiotics), the patient's CRP fell from 6 to $2 \mathrm{mg} / \mathrm{L}$. Once the end point of CRP $\leq 2 \mathrm{mg} / \mathrm{L}$ was reached, the sternal deficit was reconstructed using porcine $\mathrm{ADM}$, which was soaked in rifampin. The wound healed without further complications, demonstrating key benefits of biological repair materials: adequate strength for closure of a sternal deficit and utility in the setting of a contaminated/infected wound.

In conclusion, complete congenital sternal cleft is a rare abnormality that requires closure to protect the mediastinal structures and support thoracic function. The authors utilized negative pressure therapy to stabilize pulmonary function, which facilitated weaning the patient from high-flow nasal cannula, along with direct antibiotic wound irrigation and systemic antibiotics preparing the wound for closure. Timing of wound closure was determined by CRP level. Porcine ADM was then used to successfully manage absent sternum in an infant with an infected sternal surgical site previously repaired with synthetic mesh.

\section{NOTES}

\section{Conflict of interest}

No potential conflict of interest relevant to this article was reported.

\section{Ethical approval}

The study was performed in accordance with the principles of the Declaration of Helsinki. Written informed consent was obtained.

\section{Patient consent}

The patient's family provided written informed consent for the publication and the use of his images.

\section{Author contribution}

Conceptualization, writing original draft: Semlacher RA. Writing review and editing: Nuri MA.

\section{ORCID}

Roy Alfred Semlacher https://orcid.org/0000-0002-32871121

\section{REFERENCES}

1. Torre M, Rapuzzi G, Carlucci M, et al. Phenotypic spectrum and management of sternal cleft: literature review and presentation of a new series. Eur J Cardiothorac Surg 2012;41: 4-9.

2. Sarper A, Oz N, Arslan G, et al. Complete congenital sternal cleft associated with pectus excavatum. Tex Heart Inst J 2002; 29:206-9.

3. Suri RK, Sharma RK, Jha NK, et al. Complete congenital sternal cleft in an adult: repair by autogenous tissues. Ann Thorac Surg 1996;62:573-5.

4. Kohli V, Nooreyazdan S, Das BN, et al. Surgical reconstruction for absence of sternum and pericardium in a newborn. Indian J Pediatr 2006;73:367-8.

5. Al-Yamani M, Lavrand F, Thambo JB, et al. Upper sternal cleft with a complex congenital heart defect: repair in a single stage. Ann Thorac Surg 2016;101:760-2.

6. Olthof PB, Colenbrander DA, Van Der Kuip M, et al. Meshrepaired complete sternal cleft complicated by multi-resistant bacterial infection. APSP J Case Rep 2014;5:30.

7. Paton BL, Novitsky YW, Zerey M, et al. Management of infections of polytetrafluoroethylene-based mesh. Surg Infect (Larchmt) 2007;8:337-41.

8. de Vries Reilingh TS, van Goor H, Charbon JA, et al. Repair of giant midline abdominal wall hernias: "components separation technique" versus prosthetic repair: interim analysis of a randomized controlled trial. World J Surg 2007;31:756-63.

9. Szczerba SR, Dumanian GA. Definitive surgical treatment of infected or exposed ventral hernia mesh. Ann Surg 2003;237: 437-41.

10. Diaz JJ Jr, Guy J, Berkes MB, et al. Acellular dermal allograft for ventral hernia repair in the compromised surgical field. 
Am Surg 2006;72:1181-7.

11. Ventral Hernia Working Group, Breuing K, Butler CE, et al. Incisional ventral hernias: review of the literature and recommendations regarding the grading and technique of repair. Surgery 2010;148:544-58.

12. Gupta S. Optimal use of negative pressure wound therapy for skin grafts. Int Wound J 2012;9 Suppl 1:40-7.

13. Atkins BZ, Tetterton JK, Petersen RP, et al. Laser Doppler flowmetry assessment of peristernal perfusion after cardiac surgery: beneficial effect of negative pressure therapy. Int
Wound J 2011;8:56-62.

14. Colli A, Camara ML. First experience with a new negative pressure incision management system on surgical incisions after cardiac surgery in high risk patients. J Cardiothorac Surg 2011;6:160

15. Ennker IC, Malkoc A, Pietrowski D, et al. The concept of negative pressure wound therapy (NPWT) after poststernotomy mediastinitis: a single center experience with 54 patients. J Cardiothorac Surg 2009;4:5. 